\title{
The activation strain model in the light of real space energy partitions ${ }^{\dagger}$
}

\author{
José Luis Casals-Sainz, Evelio Francisco, Ángel Martín Pendás ${ }^{\text {aa }}$ \\ January 30, 2020
}

\begin{abstract}
The distortion/interaction or activation strain model (ASM) of chemical reactivity is examined in real space through the interacting quantum atoms (IQA) approach. Attention is paid to the role that the geometrically constrained ASM structures of the fragments play in the chemical interpretation of the driving forces that lead to a given reaction channel. These fictitious intermediate states are necessary in the ASM, but IQA may or may not use them at will. Similarities and differences are highlighted by studying the endo/exo preference rules of simple [4+2] Diels-Alder cycloadditions. Although overall the agreement is reasonable, we warn about a blind use of the plain ASM if no further energy decomposition analyses of its interaction energy are done.
\end{abstract}

Keywords: Quantum chemistry; Computational chemistry; Energy decomposition analysis; Interacting quantum atoms method.

${ }^{a}$ Departamento de Química Física y Analítica, Facultad de Química, Universidad de Oviedo. 33006 Oviedo, Spain. email: ampendas@uniovi.es 


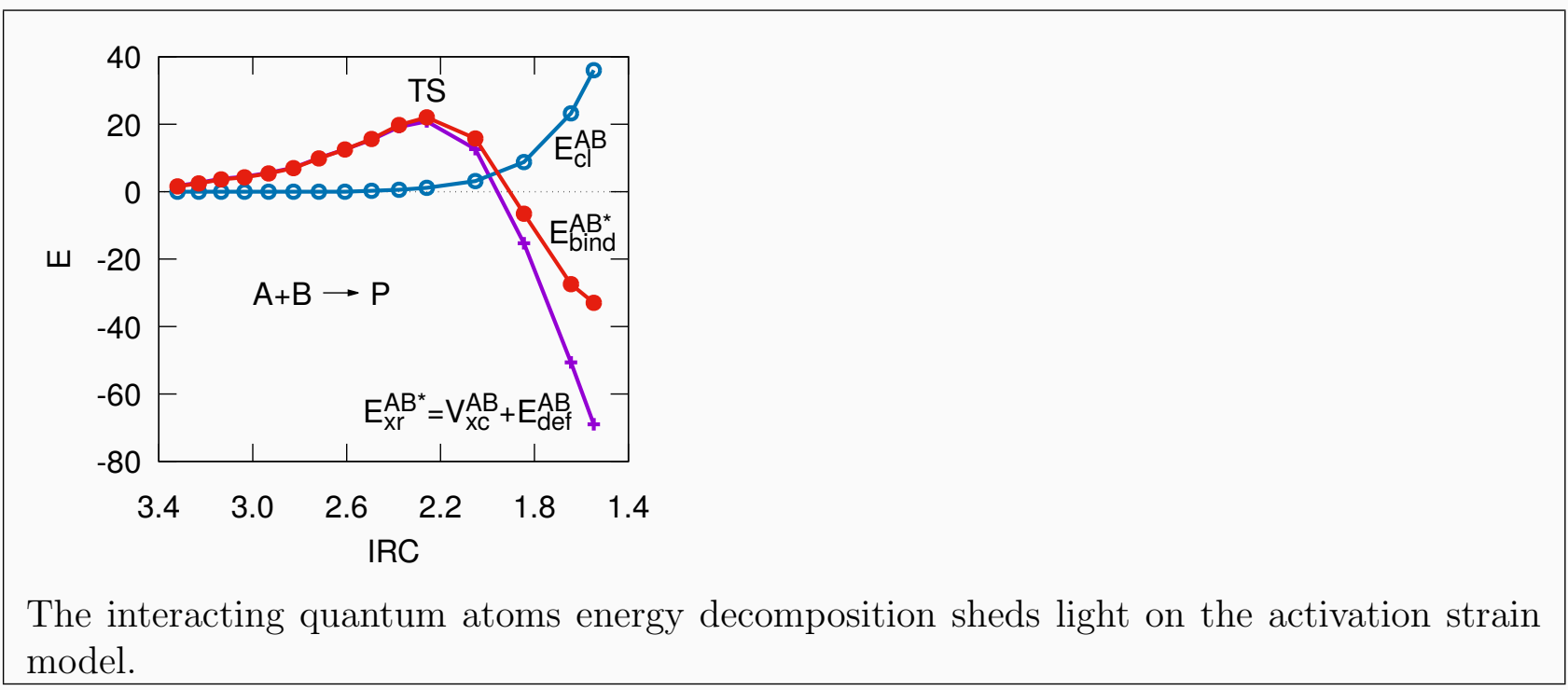




\section{INTRODUCTION}

Chemical reactivity, as well as chemical bonding theory, have been developed over the years following several non-necessarily compatible frameworks. In one, the focus is put on the (re)distribution of electrons as a process leading to the formation and/or breaking of some bonds, or in a particular reaction channel that takes place according to the laws of quantum mechanics. In this view, symmetry preservation rules, one-particle descriptors such as orbital energies and electron counting parameters like bond orders, but also scalar fields like the electron density, its laplacian, or the electron localization function (ELF), are used to build predictive insights on how molecules form or rearrange. The total energy, however, is not an intrinsic part of these models, although (in some of them) energy changes can be approximated through perturbation theory, for instance. This standpoint has led, to cite just a few, to the Woodward-Hoffmann rules, ${ }^{[1]}$ the frontier molecular orbital (FMO) theory of Fukui, ${ }^{[2]}$ the natural bond order (NBO) analysis of reactivity, ${ }^{[3]}$ or the bond evolution theory $(\mathrm{BET}) \cdot{ }^{[4-7]}$

Another route focuses on the energy itself and its evolution from reactants to products. This includes plain intrinsic reaction coordinate (IRC) analyses of potential energy surfaces, the Marcus theory, ${ }^{[8]}$ and the valence-bond curve-crossing model, and also deeper methods like conceptual density functional theory $(\mathrm{CDFT})^{[9]}$ that, although perturbative in nature, have provided much insight on the roots of a large number of quasi-empirical rules in chemical

reactivity like the principle of equalization of electronegativities ${ }^{[10,11]}$ or the hard soft acid base (HSAB) principle. Digging into the nature of the energy changes accompanying a chemical process usually calls for an energy decomposition analysis (EDA). This must be valid at any point of the IRC, leaving out accurate and insightful modern perturbation approaches like symmetry adapted perturbation theory (SAPT) ${ }^{[12]}$. In many cases, the intrinsic descriptors and language of the chosen EDA are incompatible, or independent to say the least, from those used in the models described in the previous paragraph, leading to an uncoupling of both kinds of methodologies.

In the last years, a particular application of EDAs to the field of chemical reactivity, known as the distortion/interaction or the activation strain model (ASM) has made its 
way. ${ }^{[13-15]}$ Succinctly, the ASM recognizes the role of geometrical deformation (strain, distortion) on building the activation barrier at the transition state (TS) of a bimolecular chemical reaction. According to the ASM, the energy of the TS is determined not only by how the reactants interact with each other, but also by the energy penalty due to the strain that leads to the TS geometry. If a fragment needs to deform non-negligibly to acquire the TS structure, the barrier is expected to increase, for instance. In the ASM, the barrier is simply a balance between the necessarily destabilizing distortion energy of the fragments and their mutual interaction. Up to now, the ASM has been successfully applied to substitutions, eliminations, pericyclic processes, and organometallic reactions. ${ }^{[14,15]}$

There are two basic ingredients in the ASM besides an appropriate reaction coordinate $\xi$ : the energies of the isolated fragments at each of the strained/distorted geometries along the selected path, and an appropriate partitioning method of their mutual interaction energy. Usually, although as we are going to see, not necessarily, the ASM is used in conjunction with the extended transition state (ETS) methodology of Ziegler and Rauk, ${ }^{[16-18]}$ which builds upon Morokuma's ideas. ${ }^{[19-21]}$ This methodology was also developed by Bickelhaupt and Baerends ${ }^{[22]}$ within density functional theory, and in this latter field it is usually referred to as the energy decomposition analysis (EDA). ETS/EDA has been extensively used by several schools, including that of Frenking, ${ }^{[23,24]}$ and has been coupled to the natural orbitals for chemical valence (NOCV) method to provide an even thinner energetic partition (ETSNOCV). ${ }^{[25]}$

Intrinsic to the ASM or to the ASM+EDA procedures is the use of non-stationary states, both geometrically (to obtain the strain energy) and electronically (at the EDA orthogonalization/antisymmetrization step). This leads to several potential interpretation problems as well as to interesting epistemological debates. In this regard we recommend the many essays by W. H. Eugen Schwarz on this topic. ${ }^{[26-29]}$ In short, it is very easy to fall into conceptual pitfalls when trying to "explain" the "origin" of a given chemical behavior from quantum mechanical descriptors. To this author, description is not the same as explanation, much as correlation is not causation, and the use of intermediate states is essential, different choices leading to complementary explanations.

A relevant point that has been at the core of several debates in the near past, like that 
regarding the origin of the rotation barrier in ethane ${ }^{[30,31]}$ or, more recently, the one on the role of steric hindrance in $\mathrm{SN}_{2}$ reactions ${ }^{[32]}$ is the paradoxical behavior that internal relaxation mechanisms activated after perturbing a system impinge on quantum mechanical descriptors. In many situations, the vertical sudden change suffered by a quantity used to sense a given phenomenon after the onset of a perturbation changes sign after relaxation, i.e. it overshoots.

If chemical intuition is built upon the change of the sensing descriptor on the vertical (possibly non-stationary) state, conflicting interpretations based on methods that do or do not use intermediate states will arise. An old, yet illuminating example is the debate on the forces driving covalent bonding, as highlighted by Schwarz and Schmidbaur: ${ }^{[26] ~ " E l e c t r o n ~}$ sharing (diatomic delocalization) reduces the kinetic energy density functional, permitting orbital contraction, which raises the kinetic energy value for the benefit of a potential energy decrease." Taking the kinetic energy as an electron delocalization proxy is a perfectly valid choice. However its relaxation overshoots through the virial theorem.

Since the use of the ASM in chemical reactivity is intimately linked to geometrically strained intermediate states, the ASM is prone to the paradoxes just described. ${ }^{[26]}$ In a way, particularly near TSs where strain is large, separating geometric from electronic deformation is rather arbitrary. After all, fragments deform as a response to interactions, so charge transfer, electron delocalization, and electrostatic stabilizations/destabilizations all mingle together. In ASM+EDA, for instance, separating Pauli repulsion in the geometrically relaxed interacting fragments from strain is just one out of many possible choices.

Real space partitioning provides a route to gauging the role of deformation or strain for any number of fragments at general geometries without using non-stationary states. ${ }^{[33]}$ The latter can indeed be used if one chooses to, and this flexibility allows for fruitful comparisons among different partitioning methodologies. ${ }^{[34]}$ In the interacting quantum atoms (IQA) approach, ${ }^{[35,36]}$ for example, the energy is directly written as strain (deformation in IQA parlance) plus interaction. Even the geometric strain of a fragment can be decomposed into the atomic strains of its constituents and the change in their pairwise additive interactions. As far as we know, IQA has not yet been used in the ASM context. Thus our main objective here is to provide a first comparison, focusing on similiarities and differences between the 
two approaches.

To do so, we have chosen a relatively recent example ${ }^{[37]}$ regarding the origin of the endo rule in simple Diels-Alder (DA) reactions. The DA [4+2] cycloaddition is one of the most important pericyclic processes ${ }^{[38]}$ showing clear regio- and steroselectivity that leads, in many cases, to the preferable formation of the endo product. This preference, since formulated by Alder and Stein, ${ }^{[39]}$ has been subjected to intense theoretical scrutiny, and the solution proposed by Hoffmann and Woodward, ${ }^{40]}$ the so-called secondary orbital interactions (SOI) model that has dominated the field for years, has been challenged from several fronts. In this sense, the endo preference has been related to charge-transfer, ${ }^{[41]}$ inductive, ${ }^{[42]}$ or steric ${ }^{[43]}$ interactions, to name a few possibilities. At the root of the plethora of explanations proposed lies the energy partition problem considered above. Regarding the ASM, Fernández and Bickelhaupt proposed that it was the larger strain energy of the exo TS with respect to the endo one that justifies the rule, and not any clear difference between the interaction energies of the strained fragments. Since other works have found non-negligible interaction differences, it is interesting to explore the IQA deformations/interactions with and without the consideration of intermediate geometrically constrained states.

We have thus considered the plain cis-1,3-butadiene + ethylene DA reaction with the IQA approach as well as the endo/exo preference in the DA reactions between cyclopentadiene and maleic anhydride, and between cyclopentadiene and cyclopropenone. First, we briefly describe the ASM and the IQA methodology. Then, some details on the actual computations performed to study the above three reactions are given. Finally, we discuss the results obtained and give the most relevant conclusions of this work.

\section{THE ASM AND IQA METHODOLOGIES}

We devote this Section to summarize the basic features of the ASM and the IQA partition. As briefly commented in the Introduction, the activation strain or distortion/interaction model ${ }^{[13-15]}$ was proposed as a means to understand reaction barriers from computable properties of the reactants. In the ASM, the changes in the energy along a given reaction coordinate $\xi$ (typically the intrinsic reaction coordinate, $\left.\operatorname{IRC}^{[44]}\right), \Delta E(\xi)$, is decomposed into two 
terms: (i) the strain or distortion energy, $\Delta E_{\text {strain }}(\xi)$, computed as the difference between the sum of the energies of the isolated reacting fragments at the constrained geometry found at $\xi$ and the same sum when the fragments are at their isolated equilibrium geometries; (ii) the interaction energy, $\Delta E_{\text {int }}(\xi)$, the energy difference between the interacting molecular complex at $\xi$ and the sum of the energies of the isolated fragments at the $\xi$ strained geometries:

$$
\Delta E(\xi)=\Delta E_{\text {strain }}(\xi)+\Delta E_{\text {int }}(\xi)
$$

$\Delta E_{\text {strain }}(\xi)$ is destabilizing, since the fragments move away from equilibrium when reacting, while $\Delta E_{\text {int }}(\xi)$ is usually stabilizing, taking into account all bonding interactions that will eventually lead to the products. The ASM can be successfuly used to rationalize changes in the position and energy of the TS. ${ }^{[14]}$ For instance, it is clear that if two reactions proceed with the same $\Delta E_{\text {strain }}(\xi)$, the one with larger (more stabilizing) $\Delta E_{\text {int }}(\xi)$ will display an earlier TS with smaller $\Delta E^{\ddagger}$. Similarly, at constant interaction energy, a larger strain will lead to a later TS with larger $\Delta E^{\ddagger}$. Not recognizing the $\xi$ evolution by performing a single point analysis of the strain and interaction components at the TS only may easily lead to the paradoxes already described.

$\Delta E_{\text {int }}$ is usually partitioned through the ETS/EDA technology. To that end, the two (strained) fragments' densities are allowed to interact electrostatically, then, their noninteracting wave functions are antisymmetrized and orthogonalized, and, finally, the full wave function is allowed to relax towards the finally stationary state. By doing so,

$$
\Delta E_{\text {int }}=\Delta E_{\text {elst }}+\Delta E_{\text {Pauli }}+\Delta E_{\text {orb }}
$$

The first term is the electrostatic interaction (of Pauli violating states) between the fragments, the second is associated to the Pauli repulsion, and the third to the orbital interaction. If dispersion is relevant, a $\Delta E_{\text {disp }}$ is also added to $\Delta E_{\text {int }}$.

Notice that the separation of $\Delta E_{\text {strain }}$ is done ad hoc, and that many times the strain energy is associated with steric hindrance, a term that is also linked to $\Delta E_{\text {Pauli }}$ or to $\Delta E_{\text {elst }}+\Delta E_{\text {Pauli }}$, depending on the context. This is the origin of some recent interesting controversies. ${ }^{[32,45]}$ A point on non-stationary states is also due. As it is now clear, two different sets of intermediate states are used in the ASM+EDA technology: the strained 
isolated fragments, on the one hand, and the antisymmetrized non-stationary wave function that is built from the strained fragments to get $\Delta E_{\text {Pauli }}$.

At variance with this approach, a partition of the energy that needs not include any non-stationary state is available in real space. This is offered at the cost of defining 3D regions associated to atoms or fragments, so that the arbitrariness now moves on to how an atom-in-a-molecule or a fragment-in-a-complex is built. Among the many proposed solutions to this problem, one with deeper physical roots than many others is provided by the Quantum Theory of Atoms in Molecules (QTAIM) formulated by Bader and coworkers. ${ }^{[46]}$ The only fully orbital invariant energy decomposition compatible with it at arbitrary molecular geometries is the interacting quantum atoms approach (IQA). ${ }^{[35,36]}$

In IQA we decompose the total electronic energy $(E)$ of a system into intra- and interatomic components by using the first (1-RDM) and (diagonal) second order (2-RDM) reduced density matrices of the system, which are fully orbital invariant: ${ }^{[35,36]}$

$$
E=\sum_{A} E_{\mathrm{net}}^{A}+\sum_{A>B} E_{\mathrm{int}}^{A B}
$$

where $E_{\text {net }}^{A}$ denotes the intra-atomic energy of region $A$ and $E_{\text {int }}^{A B}$ corresponds to the interaction energy between domains $A$ and $B . E_{\text {net }}^{A}$ is obtained as

$$
E_{\mathrm{net}}^{A}=T^{A}+V_{\mathrm{ne}}^{A A}+V_{\mathrm{ee}}^{A A}
$$

with $T^{A}, V_{\mathrm{ne}}^{A A}$ and $V_{\mathrm{ee}}^{A A}$ being the kinetic energy of atom $A$, the nucleus-electron interaction and the electron-electron repulsion within atom A, respectively. Similarly, $E_{\mathrm{int}}^{A B}$ stands for the sum of the nucleus-nucleus $\left(V_{\mathrm{nn}}^{A B}\right)$ and the electron-electron $\left(V_{\mathrm{ee}}^{A B}\right)$ repulsions, together with the attraction between the electrons located in atom $A$ and the nucleus in atom $B$ $\left(V_{\mathrm{ne}}^{B A}\right)$ and vice versa $\left(V_{\mathrm{ne}}^{A B}\right)$,

$$
E_{\mathrm{int}}^{A B}=V_{\mathrm{nn}}^{A B}+V_{\mathrm{ee}}^{A B}+V_{\mathrm{ne}}^{A B}+V_{\mathrm{ne}}^{B A} .
$$

One attractive feature of IQA is the fact that it is based on orbital-invariant scalar functions, which enables the separation of $E$ in terms corresponding to atoms and to atomic pairs in an unambiguous and transparent manner. Another important feature of the IQA methodology is its invariance with respect to the union of sets of atoms to form groups or 
fragments inasmuch as they cover all of the atoms of the system. By doing so, we just need to change the way we refer to labels, so that $A, B$ will now refer to molecular fragments instead of atoms. Notice that the net (or self) energy of a fragment now contains the mutual interactions of all its atomic pairs. ${ }^{[47]}$

The interelectron repulsion term can be further divided into Coulombic and exchangecorrelation contributions. This division enables a rearrangement of the whole interatomic energy as a sum of classical $\left(V_{\mathrm{cl}}^{A B}\right)$ and exchange-correlation $\left(V_{\mathrm{xc}}^{A B}\right)$ contributions,

$$
E_{\mathrm{int}}^{A B}=V_{\mathrm{cl}}^{A B}+V_{\mathrm{xc}}^{A B}
$$

$V_{\mathrm{xc}}^{A B}$ and $V_{\mathrm{cl}}^{A B}$ are identified with the covalent and ionic components of the interaction between atoms (or fragments) $A$ and $B .{ }^{[48]}$ The net energy of a fragment $X, E_{\text {net }}^{X}$, can be measured with respect to a given reference $E_{\text {net,ref }}^{X}$ This allows us to define the so-called deformation energy of this fragment as $E_{\text {def }}^{X}=E_{\text {net }}^{X}-E_{\text {net,ref }}^{X}$, so that the binding energy of the complex with respect to those references is given by

$$
E_{\text {bind }}^{A B}=E_{\text {int }}^{A B}+E_{\text {def }}^{A B}
$$

where $E_{\mathrm{def}}^{A B}=E_{\mathrm{def}}^{A}+E_{\mathrm{def}}^{B}$. If, as usual, the reference energy state of each fragment is taken as that of its isolated equilibrium structure, $E_{\text {def }}^{X}$ can be split into the strain enery leading to its structure at each $\xi$ and the purely electronic deformation that takes it to the in-the-molecule state,

$$
E_{\text {def }}^{X}=E_{\text {def,geom }}^{X}+E_{\text {def,el }}^{X},
$$

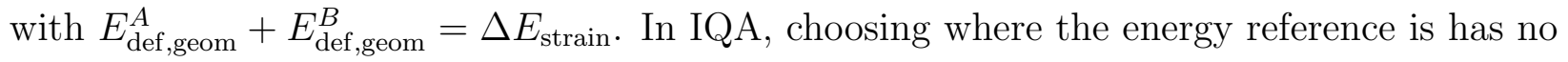
influence at all on its dynamic descriptors $\left(E_{\mathrm{net}}, V_{\mathrm{xc}}, V_{\mathrm{cl}}\right)$. Thus, the decision whether to split or not to split the deformation energy into strain plus electronic components is a matter of taste. This is not so in the ASM, since modifying the reference affects both the strain and the interaction energies.

A few words of caution are due. An orthodox IQA decomposition rests on the nondiagonal 1-RDM and the diagonal 2-RDM, ${ }^{[35,36]}$ and both are actually not available in DFT. However, approximations to by-pass these problems have been proposed. In one approach, [49] all the exchange-correlation energy not accounted for by the Kohn-Sham Fock-like exchange 
is assigned to the net energy. This has been shown to overestimate systematically the exchange-correlation component of $V_{\mathrm{ee}}^{\mathrm{AA}}$ in detriment of $E_{\mathrm{int}}^{\mathrm{AB}}$. In a second proposal that alleviates this problem, some of us have used scaling arguments to introduce also correlation in the interatomic terms. ${ }^{[50]}$

\section{COMPUTATIONAL DETAILS}

We have examined both the plain cis-1,3-butadiene plus ethylene DA reaction to show how the ASM and IQA methodologies relate to each other as well as the cycloadditions between cyclopentadiene and maleic anhydride or cyclopropanone to study the fulfilment or violation of the endo rule. For instance, when cis-1,3-butadiene reacts with maleic anhydride, only the least thermodynamically stable endo product is formed thanks to an experimental $\Delta \Delta G^{\ddagger}=2.5 \mathrm{kcal} / \mathrm{mol}$ at $298 \mathrm{~K}$ between the exo and endo TSs. ${ }^{[51]}$

We have proceeded as follows. Firstly, the transition state was found for each reaction, ensuring that it only had one imaginary frequency; then, the reaction path was followed using the IRC method. This procedure was done at the aug-cc-pVDZ ${ }^{[52]} /$ B3LYP $^{[53,54]}$ level with the GAMESS-US code. ${ }^{[55]}$ The role of dispersion energy in these reactions has been shown to be small, ${ }^{[37]}$ so no dispersion correction has been included. In this way, all IQA descriptors are clean. To simplify as much as possible, some points along the IRCs have been excluded, for instance, those including the trans to cis isomerization step of the diene or the initial van der Waals reactive complexes.

Subsequent analyses were performed at the def2-QZVPPD ${ }^{[56]}$ level using the density fitting technique with the corresponding auxiliary def2-QZVPP/JKFIT ${ }^{[57]}$ basis set with the B3LYP functional and the standard Becke grid ${ }^{[58]}$. Instead of the usual Bragg radii, we have used the covalent radii proposed in Ref. 59, using and ultra fine grid to ensure saturation and to avoid problems with rotational invariance and possible innacuracies. All these calculations were done with PySCF. ${ }^{[60]}$

The in-house program promolden ${ }^{[61]}$ was used to obtain the IQA energy terms. IQA integrations were performed using $\beta$-spheres. Restricted angular Lebedev quadratures with 3074 points and 351 points Gauss-Chebyshev mapped radial grids were used inside the 

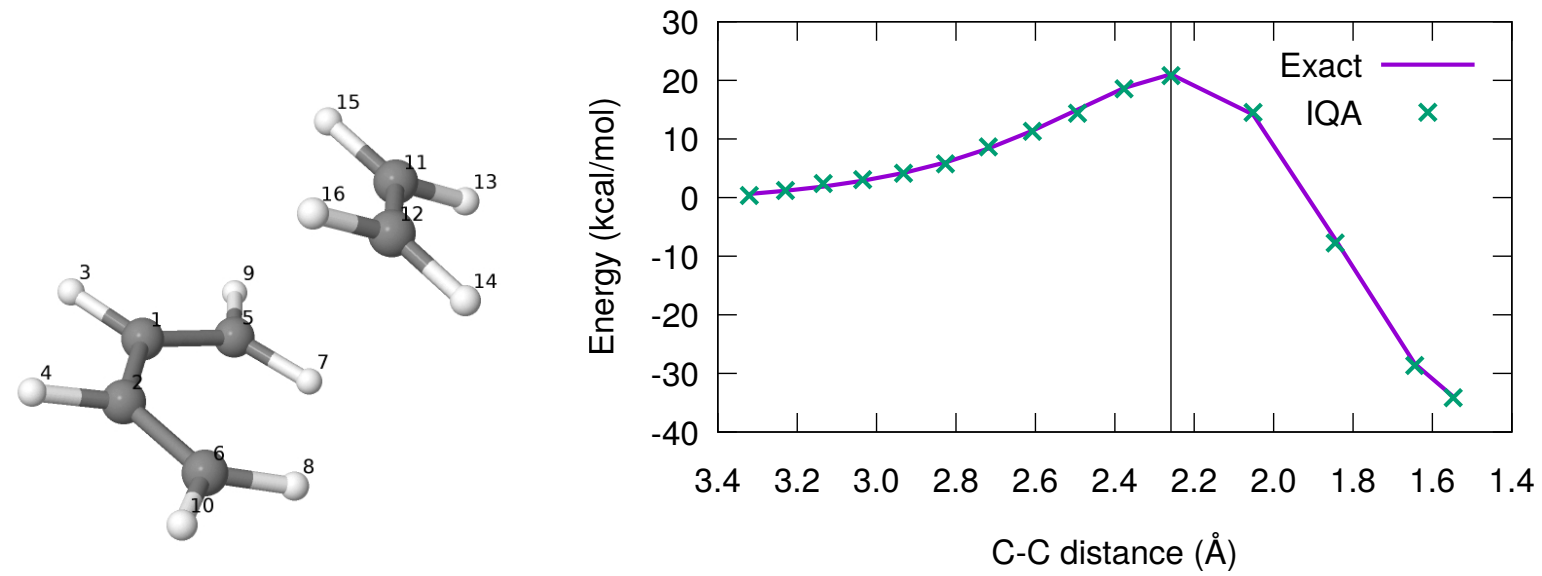

Figure 1: Numbering of atoms (left) and total energy profile along the IRC (right) for the reaction of cis-1,3-butadiene (A) with ethylene (B).

$\beta$-spheres, with $L$ expansions cut at $l=6$. Outside the $\beta$-spheres, extended 5810-point Lebedev, 551 mapped radial point Gauss-Legendre quadratures, and $L$ expansions up to $l=10$ were selected. The accuracy of the reconstructed energies are below $0.6 \mathrm{kcal} / \mathrm{mol}$.

\section{RESULTS AND DISCUSSION}

\section{The DA reaction between cis-1,3-butadiene and ethylene}

We have projected the IRC onto the $\mathrm{C}-\mathrm{C}$ distance $(\mathrm{R})$ of any of the two single bonds that are formed during the cycloaddition of cis-1,3-butadiene and ethylene. In the following, we will denote the diene (here $\mathrm{C}_{4} \mathrm{H}_{6}$ ) as fragment $A$ and the dienophile (here $\mathrm{C}_{2} \mathrm{H}_{4}$ ) as fragment $B$. The TS of the reaction is located at $R=2.26 \AA$ with an electronic $\Delta E^{\ddagger}=20.4 \mathrm{kcal} / \mathrm{mol}$. This number compares reasonably well with other DFT calculations that use the ASM as well as with the high level CCSD(T)/cc-pVTZ//B97-XD/ aug-cc-pVTZ calculation by Sexton,

Kraka and Cremer, ${ }^{[62]}$ that provides a value of $22.4 \mathrm{kcal} / \mathrm{mol}$. It suffices our purpose here. The reaction is exothermic by $34.6 \mathrm{kcal} / \mathrm{mol}$. The total energy profile along the IRC is depicted at the right part of Fig. 1.

Before considering other phenomena, it must be noticed that the real space fragments here considered are close to, but not strictly neutral, as shown in Fig 2. The sum $Q_{A}+Q_{B}$, 


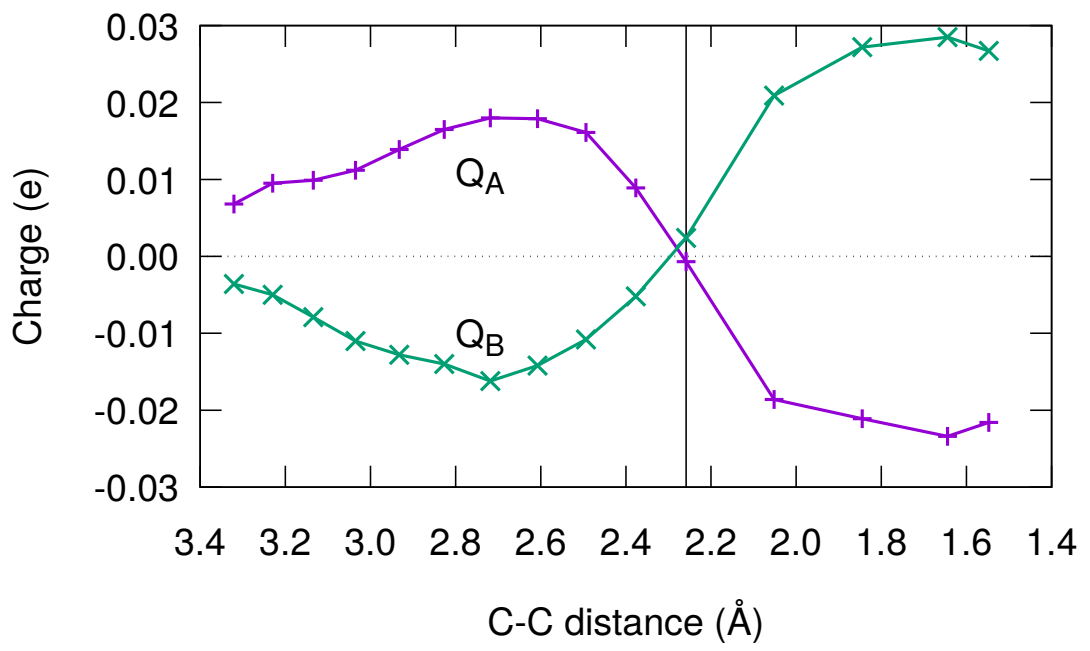

Figure 2: Total charges $\left(Q_{A}, Q_{B}\right)$ of cis-1,3-butadiene (A) and ethylene (B) along the IRC.

that should be exactly zero at any $R$, is around $\approx 0.001-0.006$, a value typical of IQA numerical integrations. At the early stages of the reaction there is a small, non-negligible electron transfer from the diene to the dienophile, compatible with the onset of delocalization between a more delocalized entity like cis-1,3-butadiene toward a more compact ethylene. As the reaction proceeds, this tendency reverses sign. At the TS the two fragments are quasi-neutral, in agreement with its purported aromatic character (which can be analyzed in real space, although we do not pursue this goal here). As we approach the products from the TS, the diene becomes negatively charged, in agreement with its larger electronegativity as a group with respect to the $\mathrm{C}_{2} \mathrm{H}_{4}$ fragment. This simple consideration shows that the interaction energy between the diene and the dienophile in-the-complex will display some kind of electrostatic term, whose energetic role is still to be determined.

The total deformation energies of the diene $\left(E_{\mathrm{def}}^{A}\right)$ and dienophile $\left(E_{\mathrm{def}}^{B}\right)$ are shown in Fig. 3, each separated into the strain component of the ASM $\left(E_{\text {def,geom }}^{A}, E_{\text {def,geom }}^{B}\right)$ and the electronic component $\left(E_{\mathrm{def}, \mathrm{el}}^{A}, E_{\mathrm{def}, \mathrm{el}}^{B}\right)$. Both strain contributions are rather small in the early stages of the reaction, increasing toward the products. In agreement with previous works, ${ }^{[14,15]}$ the strain along the full IRC is consistently larger in the diene than in the dienophile. Although the origin of this can be fully analyzed, for the sake of brevity, we just notice that this behavior is compatible with the larger number of atoms that are ge- 


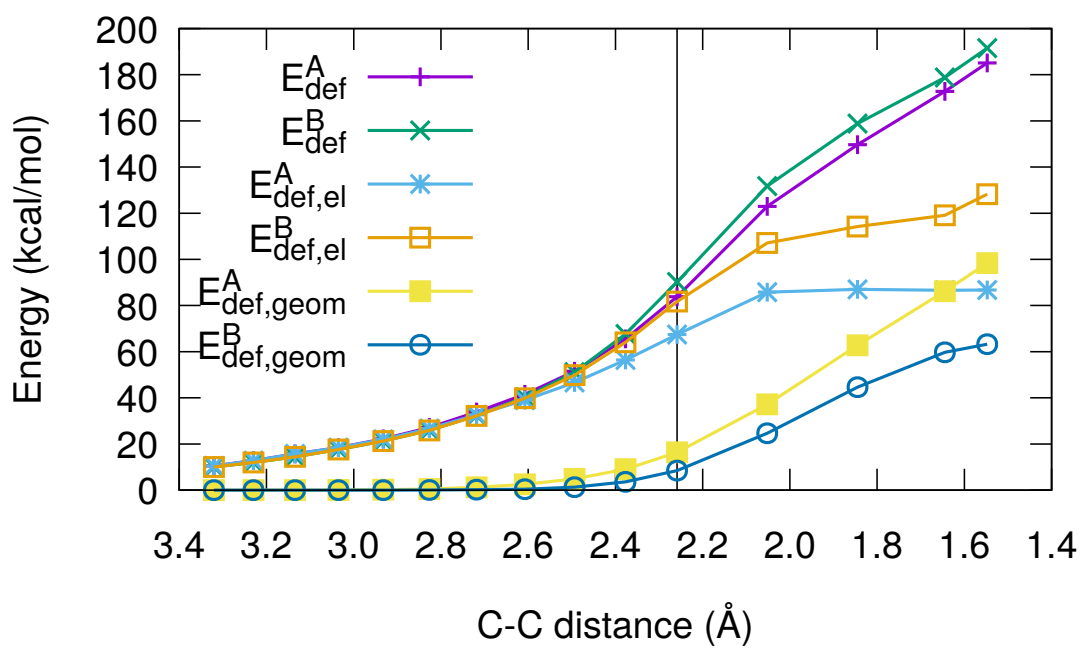

Figure 3: Deformation energies along the IRC for the reaction of cis-1,3-butadiene with ethylene.

ometrically deformed in the $A$ moiety. From these strained geometries, IQA tells us that the electronic deformation needed to take the isolated strained geometries into the in-thecomplex state are considerably larger than the geometric deformations. $E_{\text {def,el }}^{A}$ is rather close to $E_{\text {def,el }}^{B}$ in the early phases of the process, indicating simple electron delocalization between the fragments. This is corroborated by the dominance of the exchange-correlation energy $\left(V_{\mathrm{xc}}^{A B}\right)$ in the IQA interaction energy (vide infra). However, as we approach the TS the electronic deformation of the ethylenic moiety becomes considerably larger than that of the diene. This is in part justified by the evolution of $Q_{A}$ and $Q_{B}$. The latter fragment becomes increasingly depopulated from electrons, and its self-energy is destabilized. The counterpart anionization of $A$ also rationalizes why $E_{\text {def,el }}^{A}$ ceases to increase shortly after the TS. Altogether, the total IQA $E_{\mathrm{def}}^{X}$ is larger for $B$ than for $A$. Since by incorporating not only geometric but also electronic degrees of freedom, the total IQA deformation is, in our opinion, a better measure of strain than the $A S M \Delta E_{\text {strain }}$, this simple argument shows that explanations based on plain ASM strains should be used with care.

Fig. 4 shows the full IQA interatomic energy terms taking strained and free fragment references. Only the binding energy $E_{\text {bind }}^{\mathrm{AB}}$ and exchange-repulsion energy (see below), directly affected by deformations, become modified on changing the reference, as previously stated. 


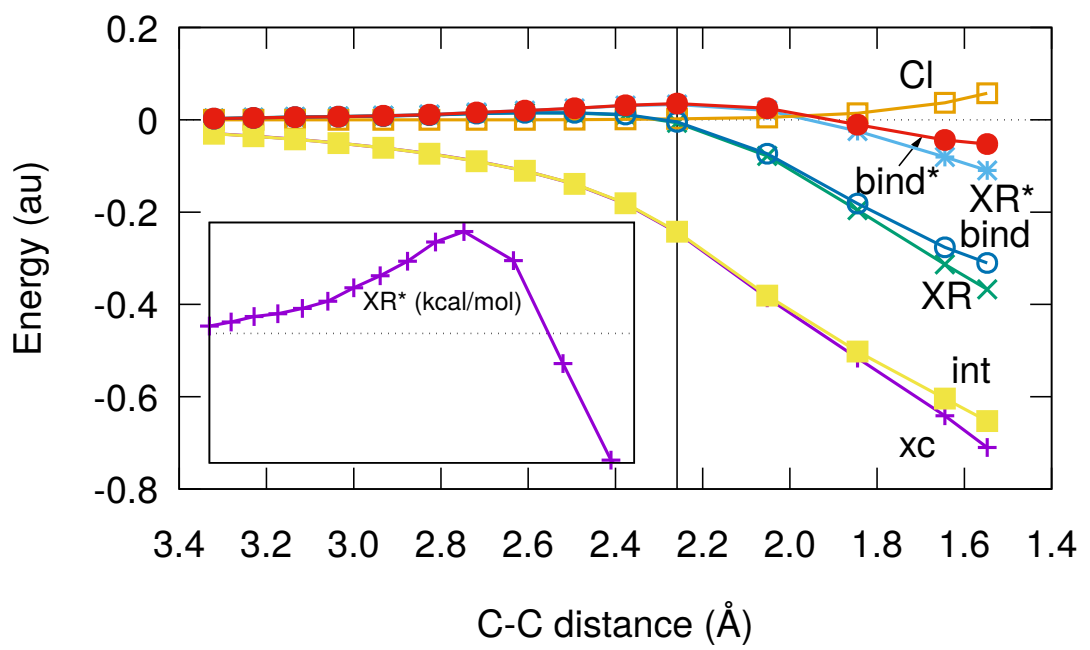

Figure 4: IQA interatomic energies (au) between cis-1,3-butadiene (A) and ethylene along the IRC. The $\mathrm{XR} \equiv E_{\mathrm{xr}}^{A B}$ and bind $\equiv E_{\text {bind }}^{A B}$ terms take only into account the electronic contributions to the deformation energies $\left(E_{\mathrm{def}, \mathrm{e}}^{A}, E_{\mathrm{def}, \mathrm{el}}^{B}\right)$ while $\mathrm{XR} \star \equiv E_{\mathrm{xr}}^{A B \star}$ and bind $\star \equiv$ $E_{\text {bind }}^{A B \star}$ take into account the full deformation energies $\left(E_{\text {def }}^{A}, E_{\text {def }}^{B}\right)$.

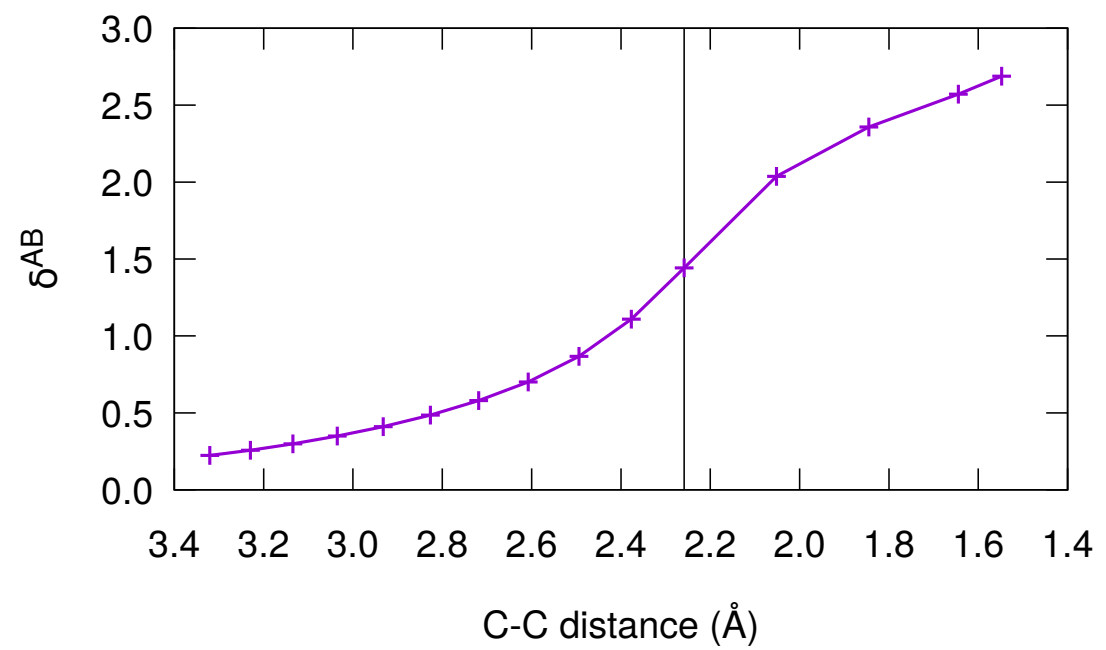

Figure 5: Delocalization index $\delta^{\mathrm{AB}}$ along the IRC for the reaction of cis-1,3-butadiene (A) with ethylene (B). 
The profiles are easily recognizable in the IQA community as coming from the onset of covalent interactions. The IQA interaction energy (not to be confused with the ASM one, which includes also Pauli deformations) is clearly dominated by exchange-correlation terms, and only in the latest steps, close to the products, does a measurable electrostatic destabilization play a role. Indeed, although charge transfer is non-zero, the overall interaction between the non-overlapping fragment densities is globally destabilizing.

An interesting point regards the so-called exchange-repulsion ${ }^{[63,64]}$ IQA energy, defined as $\mathrm{XR} \equiv E_{\mathrm{xr}}^{A B}=V_{\mathrm{xc}}^{A B}+E_{\mathrm{def}, \mathrm{el}}^{A B}\left(\right.$ or $\mathrm{XR} \star \equiv E_{\mathrm{xr}}^{A B \star}=V_{\mathrm{xc}}^{A B}+E_{\mathrm{def}}^{A B}$ if the total geometric plus electronic deformation energies are included). This quantity plays an equivalent role to its counterpart in SAPT. When electrostatic interactions are not relevant (as in this

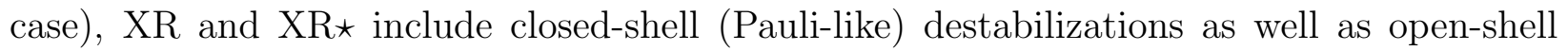
covalent stabilization. The close similarity between XR` (see inset in Fig. 4) and the IRC profile (Fig. 1, right) speaks about a gross picture in which deformations lead the barrier formation.

The electron delocalization just sketched behaves as expected for a concerted process. Fig. 5 shows that the delocalization index ${ }^{[65]}(\delta$, DI) between the two fragments evolves sigmoidally, showing an inflection point close to the TS. Sigmoidal DI shapes have been shown ${ }^{[66]}$ to denote standard covalent bond formation. Similarly, different fragmentations shown in Fig. 6 are also in agreement with the naïve concerted electron flow taught with arrow pushing techniques.

\section{The DA reaction between cyclopentadiene and maleic anhydride}

The numbering of atoms and the total energy of this reaction along the IRC for the endo and exo pathways are shown in Fig. 7. The endo reaction proceeds with and activation barrier of $17.50 \mathrm{kcal} / \mathrm{mol}$ with a distance between the active carbon atoms of $2.21 \AA$. The energy difference between the reactants and products is $13.11 \mathrm{kcal} / \mathrm{mol}$. The exo reaction has a slightly earlier transition state with $\Delta E^{\ddagger}=18.65 \mathrm{kcal} / \mathrm{mol}$ and a C-C distance of $2.23 \AA$. Now, the exothermicity is $13.87 \mathrm{kcal} / \mathrm{mol}$, showing that the exo product is thermodynami-

cally favored. Our approximate $\Delta \Delta E^{\ddagger}=1.15 \mathrm{kcal} / \mathrm{mol}$ favors a kinetically controlled endo pathway. 

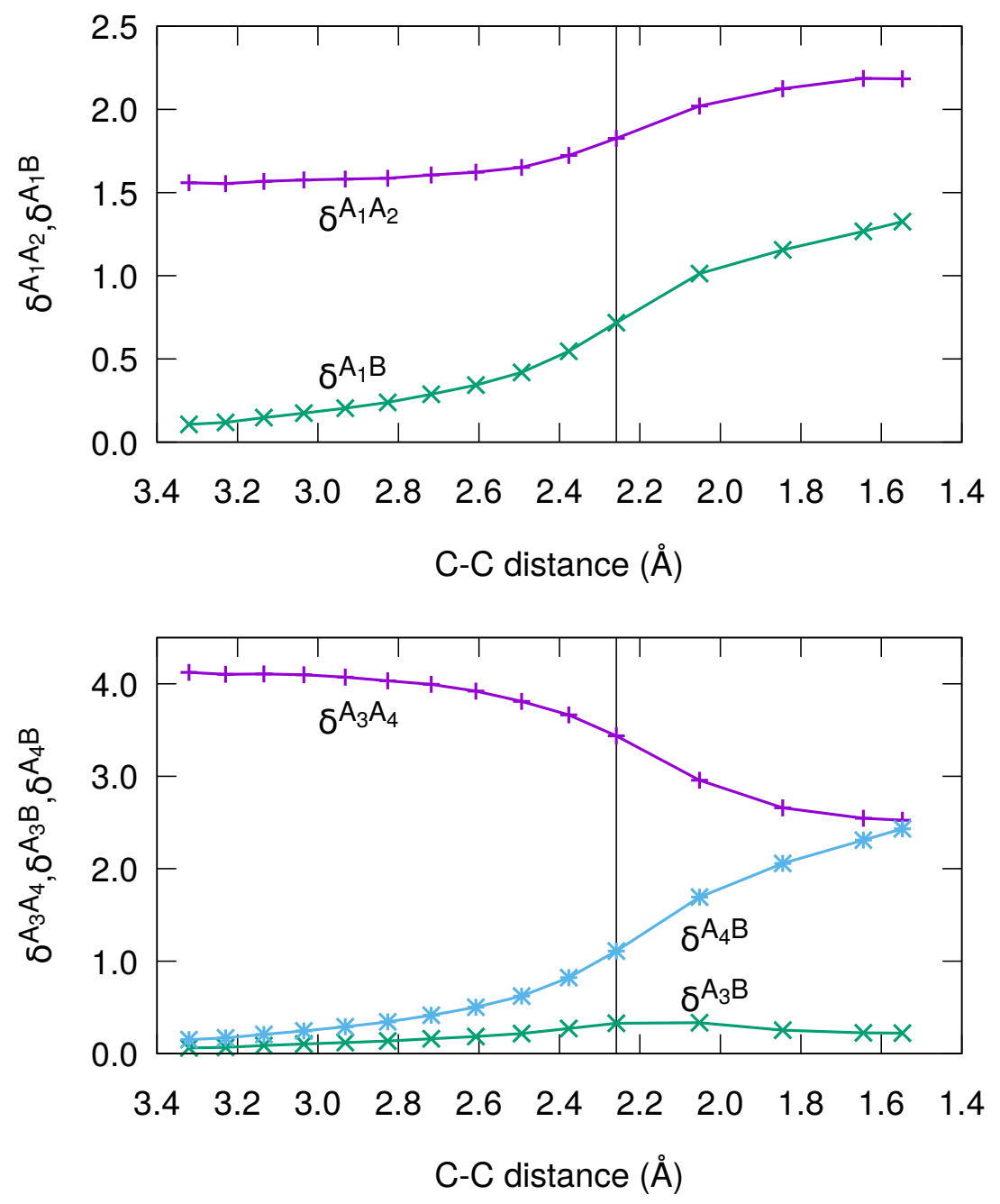

Figure 6: Delocalization indexes along the IRC of two fragmentacion schemes of cis-1,3butadiene (A). A is partitioned into $A_{1}=\{1,3,5,7,9\}$ plus $A_{2}=\{2,4,6,8,10\}$ in the top figure, and into $\mathrm{A}_{3}=\{1,2,3,4\}$ plus $\mathrm{A}_{4}=\{5,6,7,8,9,10\}$ in the bottom figure. In both figures, B corresponds to the ethylene molecule, formed by atoms 11,12,13,14,15, and 16 . 

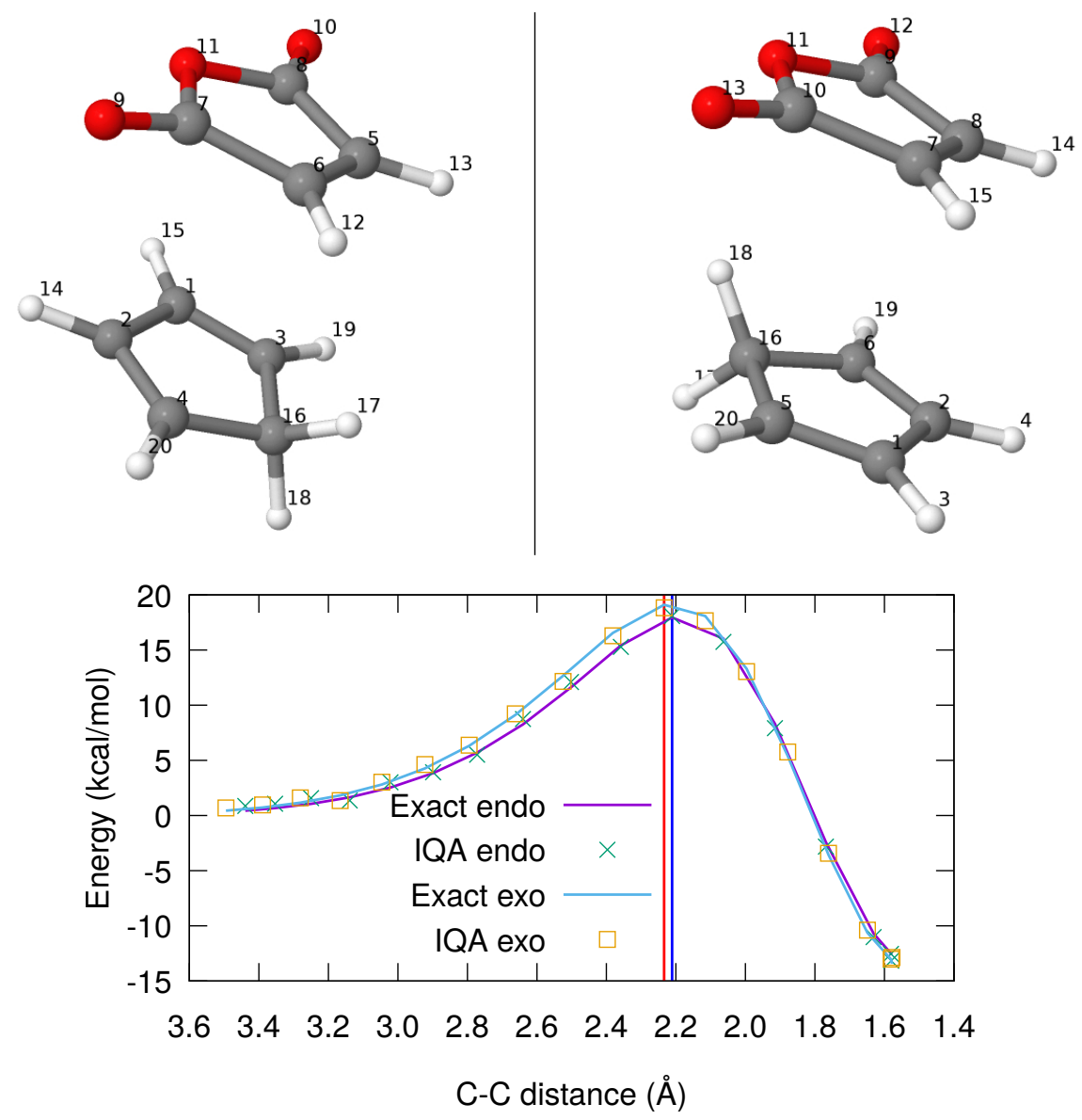

Figure 7: Endo (top-left) and exo (top-right) geometries at the beginning of the cyclopentadiene + maleic anhydride cycloaddition IRC. The total energy along the IRC, projected onto the forming $\mathrm{C}-\mathrm{C}$ bond distance, is found in the bottom diagram, where the blue and red lines indicate the position of the endo and exo TSs, respectively. 


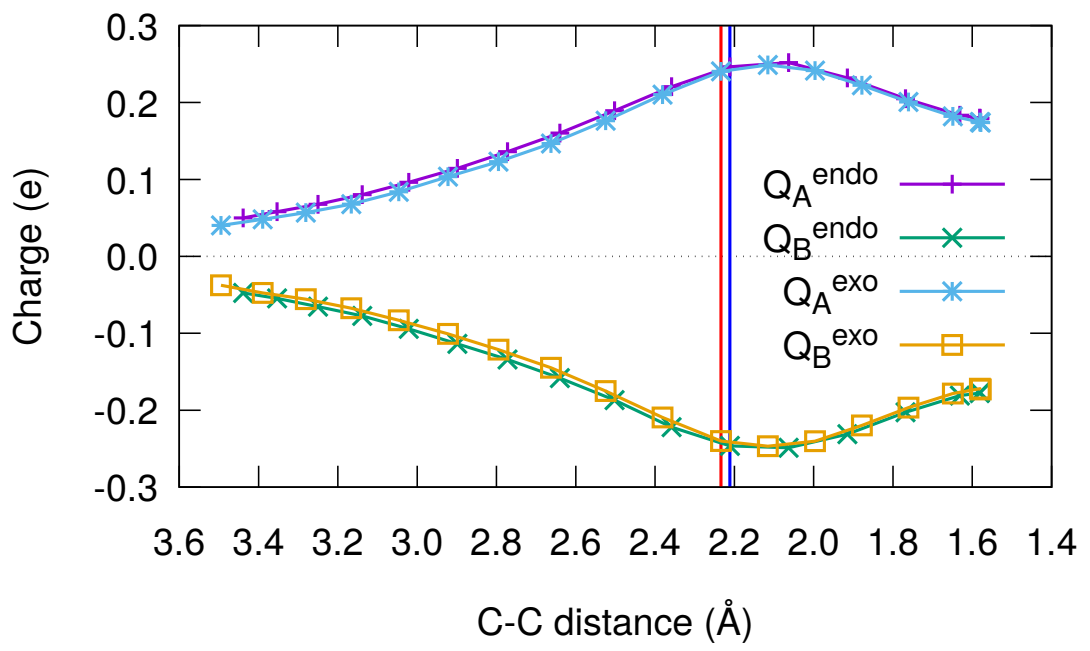

Figure 8: Total charges $\left(Q_{A}, Q_{B}\right)$ of cyclopentadiene (A) and maleic anhydride (B) along the IRC for the endo and exo reactions. The blue and red lines mark the position of the endo and exo transition states, respectively.

Fig. 8 shows the total charge of the fragments along the IRC, both for the endo and exo pathways. Contrarily to what was found in the cis-1,3-butadiene + ethylene cycloaddition, there is no charge inversion now. The reaction starts, as in the previous case, with an electron transfer from the diene to the dienophyle, which peaks significantly past the TS at about $Q_{A} \approx 0.25$. The fragments stay significantly polarized in the products, with charges close to $0.18 \mathrm{e}$. This is clearly the result of the completely different electronegativity balance. The endo pathway leads to slightly more charged fragments, particularly in the early stages of the reaction. Although this points to a more relevant role of electrostatic interactions in this case, this is not so, as we are showing.

The analysis of the deformation energy can be found in Fig. 9. As already noticed by Fernández and Bickelhaupt, ${ }^{[14]} \Delta E_{\text {strain }}$ favors the endo pathway. This is true for both the diene and the dienophile. Although it is again the diene which deforms more, the difference in strain energy between the endo and exo routes is slightly larger for the dienophile thus pointing toward steric hindrance. As in our previous example, the electronic deformation is larger than the geometric one and, contrarily to what was found there, now it is consistently greater in the $A$ fragment. The total $E_{\text {def,el }}$ is slightly larger for the endo route in the 

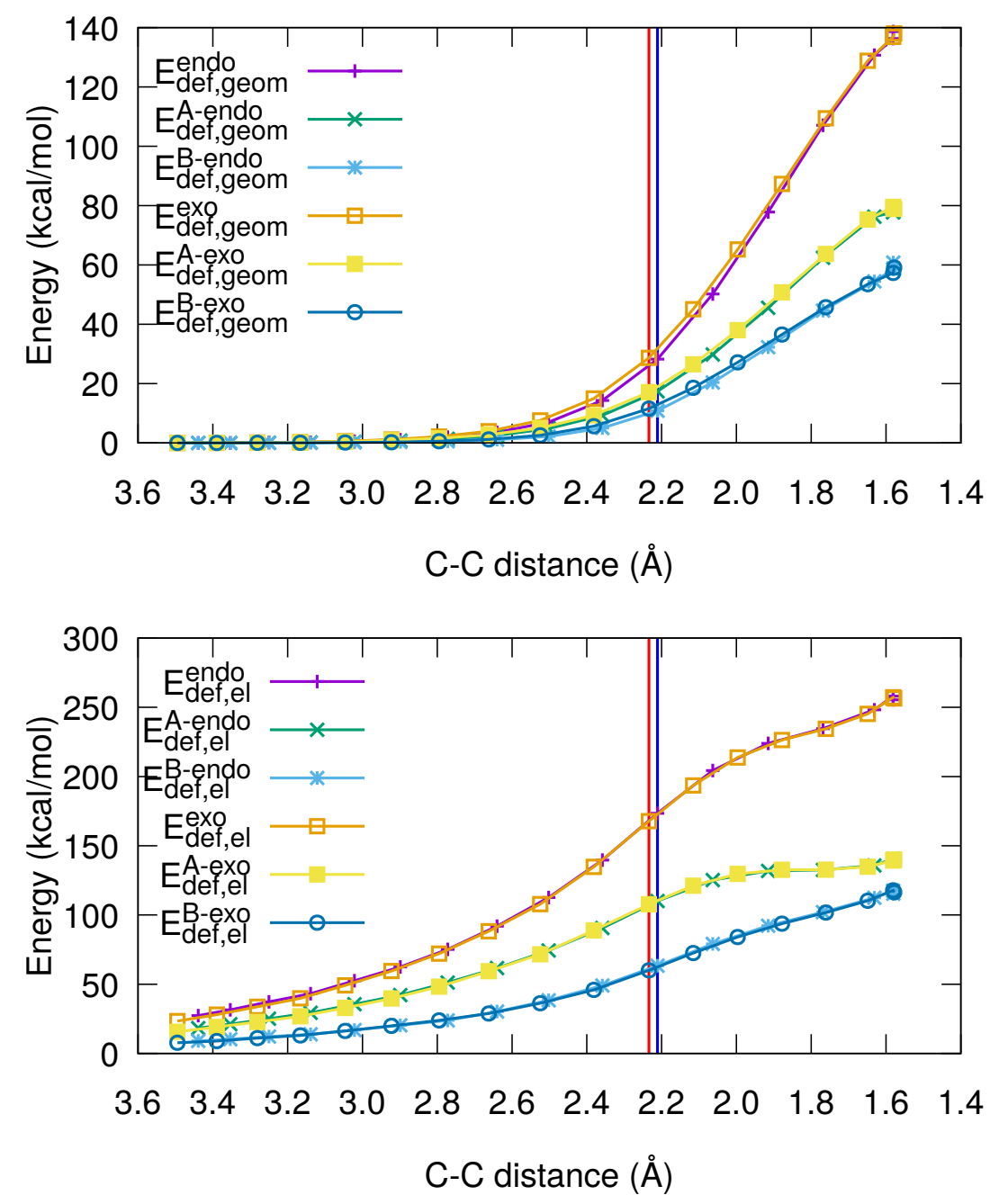

Figure 9: Geometric (geom,top) and electronic (el,bottom) deformation energies of cyclopentadiene (A) and maleic anhydride (B) along the IRC for the endo and exo reactions. The blue and red lines mark the position of the endo and exo transition states, respectively. 
earlier stages of the reaction, to become almost degenerate to that of the exo path at about $R(\mathrm{C}-\mathrm{C}) \approx 2.8 \AA$. Adding both components together, the total IQA deformation starts being larger for the endo reaction, with $\Delta \Delta E_{\text {def }}$ dominated by the electronic component in the early region, to become slightly larger for the exo route at about $R(\mathrm{C}-\mathrm{C}) \approx 2.8 \AA$, at which point $\Delta \Delta E_{\text {def }}$ is determined by the geometric factor. Notice that the larger endo electronic deformation is expected from a larger $A-B$ overlap (i.e. steric Pauli repulsion, hidden in the ASM interaction energy).

The IQA interaction energy components (Fig. 10) are also interesting. Despite the considerable charge transfer, the total electrostatic interaction is negligible except well past the TS. In the early phases of the reaction it is attractive, around $-1 \mathrm{kcal} / \mathrm{mol}$, and basically equal for the endo and exo routes. Only after the transition state it becomes destabilizing, showing that it is dominated by short-range destabilizing components. This is noteworthy, since one of the models that tries to explain the endo rule is based on the role of electrostatic interactions. Our results show that electroneutral polarizations may cancel out very efficiently, and that blind consideration of local electrostatics based on atomic or orbital charges may lead to wrong interpretations. Cancellation of short-ranged electrostatics has been analyzed recently in other contexts. ${ }^{[67]}$

$E_{\text {int }}$ is dominated by the exchange-correlation component. In agreement with what is expected from a long-range overlap, a larger $E_{\text {def,el }}$ for the endo route is accompanied by a more stabilizing endo interaction up until $R(\mathrm{C}-\mathrm{C}) \approx 2.6 \AA$ where both pathways give rise to essentially the same interaction energy. This is compatible with an early role for secondary orbital interactions $(\mathrm{SOI})^{[40]}$ that is soon lost, although a full exploration of all IQA components should be done to corroborate this claim. Since in the long-range regime, $V_{\mathrm{xc}}$ stabilizes more (usually twice as more) than what is destabilized by $E_{\text {def }}{ }^{[34]}$ the stronger early endo interaction leads to a lower energy. As we approach the TS $(R(\mathrm{C}-\mathrm{C})<2.8 \AA)$ it is the strain which drives the endo preference. Only on approaching the products does electrostatics play a non-negligible role. Overall, the binding curve (Fig. 7, bottom) is very well reproduced by the $\mathrm{XR} \star$ term (Fig. 10, top), showing that it is (it has to be) $E_{\text {def }}$ the origin of the barrier.

IQA thus provides an integrative real space perspective of the ASM. By assigning Pauli 


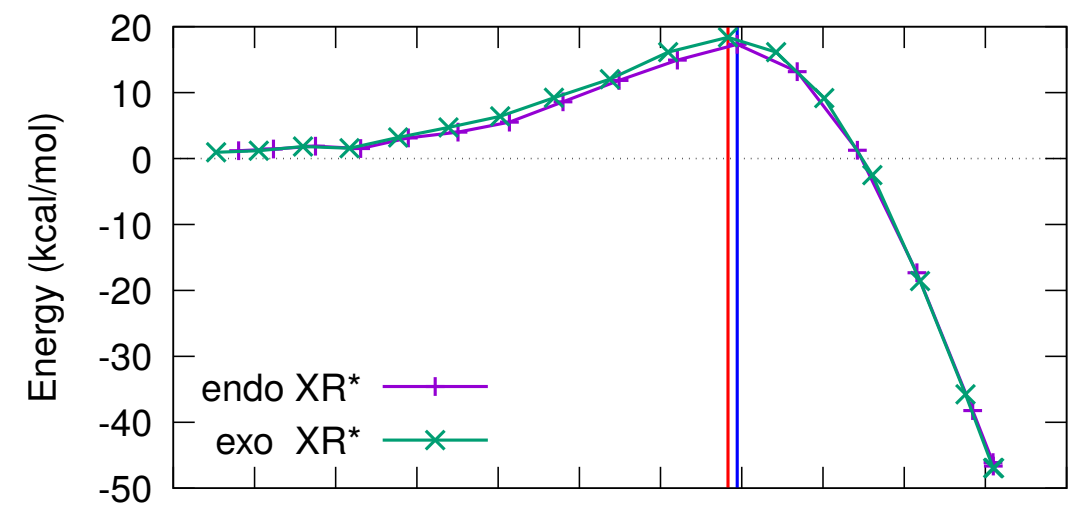

$\begin{array}{llllllllllll}3.6 & 3.4 & 3.2 & 3.0 & 2.8 & 2.6 & 2.4 & 2.2 & 2.0 & 1.8 & 1.6 & 1.4\end{array}$ C-C distance $(\AA ̊)$

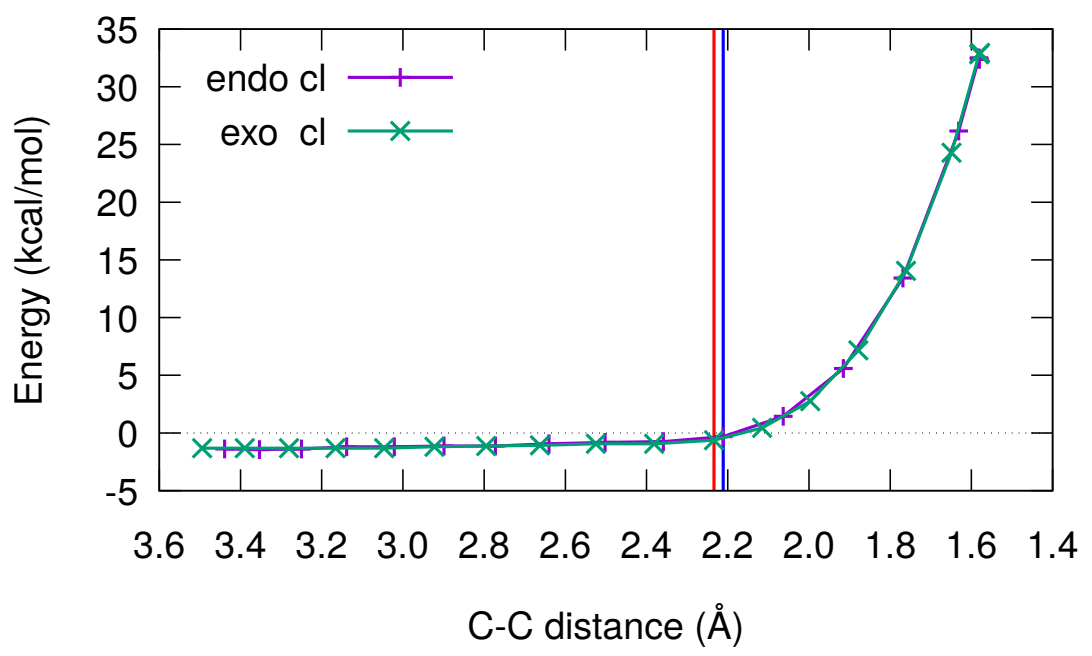

Figure 10: IQA interatomic energies $\mathrm{XR} \star=V_{\mathrm{xc}}^{A B}+E_{\mathrm{def}}^{A B}$ (top) and $V_{\mathrm{cl}}^{A B}$ (bottom) between cyclopentadiene (A) and maleic anhydride (B) along the IRC for the endo and exo reactions. The blue and red lines mark the position of the endo and exo transition states, respectively. 


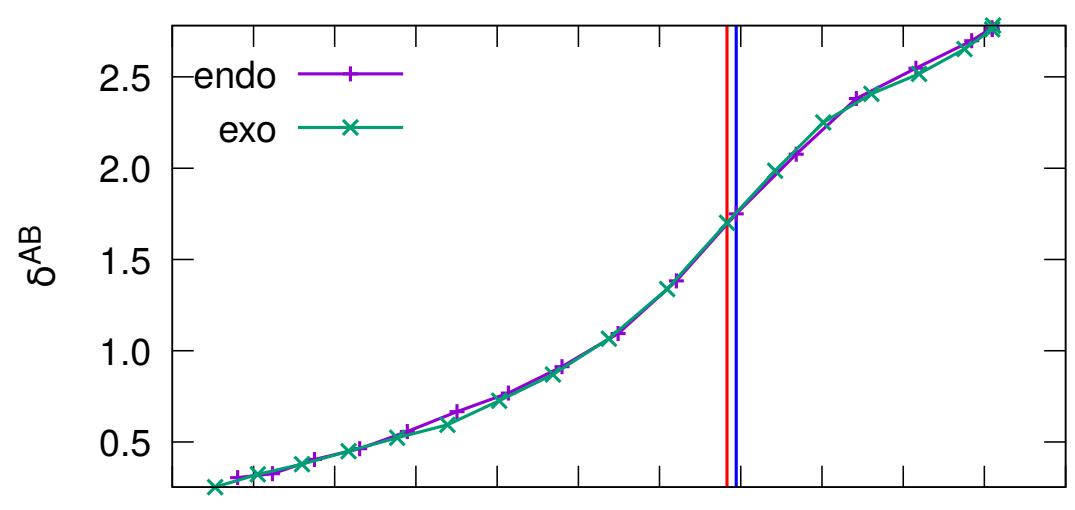

\section{$\begin{array}{llllllllllll}3.6 & 3.4 & 3.2 & 3.0 & 2.8 & 2.6 & 2.4 & 2.2 & 2.0 & 1.8 & 1.6 & 1.4\end{array}$ C-C distance $(\AA)$}

Figure 11: Delocalization index $\delta^{\mathrm{AB}}$ along the IRC for the reaction of 1,3-cyclopendadiene (A) and maleic anhydride (B) along the IRC for the endo and exo reactions. The blue and red lines mark the position of the endo and exo transition states, respectively.

and other destabilizing contributions to the interaction component of the energy, the ASM ascribes all deformations to geometry changes. As we have seen, this may lead to interpretation differences, which in the cases analyzed here are small: although electrostatic effects are basically ruled out (in these examples), a small role for SOIs is allowed both at the level of deformation and interaction in the early stages of the reaction. Notice that we could now proceed further within IQA by studying each of the interatomic interactions or atomic deformations separately. This, however lies out of the focus of the present work.

Fig. 11 shows the interfragment delocalization index along the IRC. The TSs are again rather close to the inflection point marking the onset of the newly formed covalent interactions. Notice its similarity with Fig. 5, and how the endo route leads to slightly larger values in the long-range regime.

Our results thus do support the basic claims of the ASM. However, they separate more faithfully, in our opinion, the different components that give rise to the interactions between two fragments. Notice that in a bimolecular reaction the $\mathrm{ASM} \Delta E_{\text {int }}<0$ in most cases, so that the barrier cannot be caused by anything but $\Delta E_{\text {strain }}$. An EDA decomposition of $\Delta E_{\text {int }}$ can provide further destabilizing contributions. Since in EDA $\Delta E_{\text {elst }}$ is normally stabilizing, 
the only extra positive term can only come from $\Delta E_{\text {Pauli. }}$ In this sense, $\Delta E_{\text {strain }}+\Delta E_{\text {Pauli }}$ is formally equivalent to the IQA $E_{\text {def }}$ term, although differences persist, for the IQA $V_{\mathrm{cl}}$ can be destabilizing. A comparison between EDA and IQA has already been reported. ${ }^{[34]}$

\section{The DA reaction between cyclopentadiene and cyclopropenone}

Not all DA reactions proceed through an endo pathway. This is one of the reasons why the endo rule has been revisited so many times. The cycloaddition between cyclopentadiene and cyclopropenone, for instance, prefers the exo adduct kinetically. The endo reaction displays $\Delta E^{\ddagger}=19.34 \mathrm{kcal} / \mathrm{mol}$ at a C-C distance of $2.14 \AA$ and exothermicity of $11.94 \mathrm{kcal} / \mathrm{mol}$. On the contrary, the exo reaction shows $\Delta E^{\ddagger}=17.50 \mathrm{kcal} / \mathrm{mol}$ with a $\mathrm{C}$-C distance of 2.19 $\AA$ and exothermicity of $15.84 \mathrm{kcal} / \mathrm{mol}$. This leads to $\Delta \Delta E^{\ddagger} \approx 1.83 \mathrm{kcal} / \mathrm{mol}$ favoring the exo product. Initial geometries and total energies along the projected IRC are shown in Fig. 12

The charge transfer among the fragments shows a behavior very similar to that found in the case of the maleic anhydride addition (see the supporting information), but it is now larger for the exo route in the region surrounding the TS. The behavior of deformations is peculiar, see Fig. 13. $\Delta E_{\text {strain }} \equiv E_{\text {def,geom }}$ favors the endo pathway in the early stages of the reaction. This is reversed in the vicinities of the TS, at about $R \approx 2.0 \AA$. $E_{\text {def,el }}$ is however larger throughout the IRC in the exo route. Overall, the strain dominates the endoexo preference of $E_{\text {def }}$, and the total IQA deformation favors the endo route in the pre-TS region. The IQA $V_{\mathrm{xc}}$ component is, in agreement with the $E_{\text {def,el }}$ behavior, consistently larger for the exo route along the IRC. This points to larger interfragment overlap, larger Pauli repulsion and a possible greater role of SOIs for the exo approach. The geometric strain does not correlate with the endo/exo preference in this case.

The scenario is even more interesting, since adding the total IQA deformation to $V_{x c}$ to build the $\mathrm{XR}^{*}$ term, see Fig. 14, yields a correct order and position of the TSs, but fails to reproduce the initial ordering of the exo/endo IRC energies. Surprisingly, it is the electrostatic contribution which drives the difference in this area. $V_{\mathrm{cl}}$ is stabilizing in the early phases to become destabilizing later for both routes. However, it is more stabilizing in the exo path (by about $1.7 \mathrm{kcal} / \mathrm{mol}$ ) in the long-range regime, enough so to yield the 

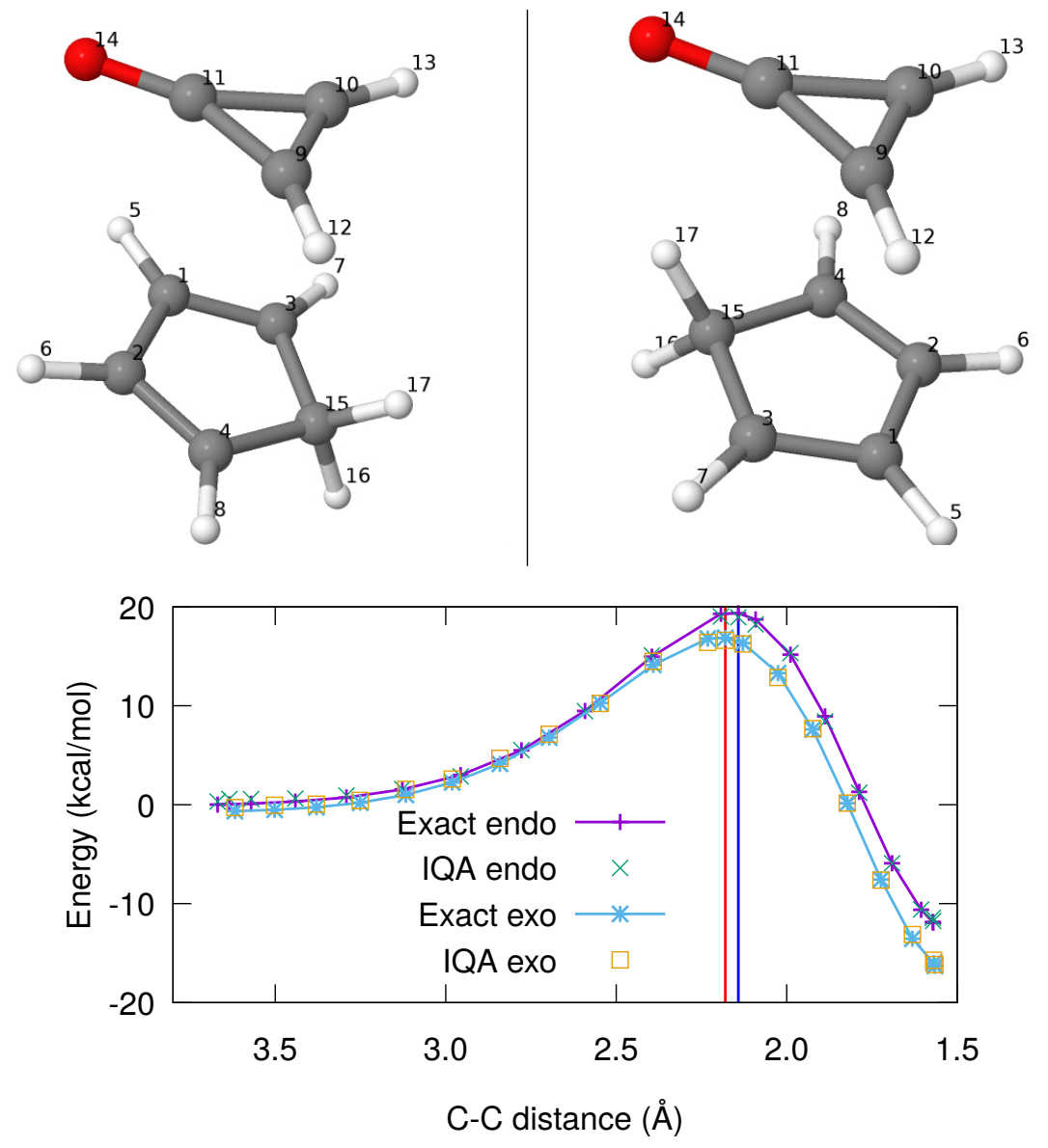

Figure 12: Endo (top-left) and exo (top-right) geometries at the beginning of the cyclopentadiene + cyclopropenone cycloaddition IRC, and total energy profile along the IRC, projected along the relevant $\mathrm{C}-\mathrm{C}$ distance (bottom). The blue and red lines mark the position of the endo and exo transition states, respectively. 


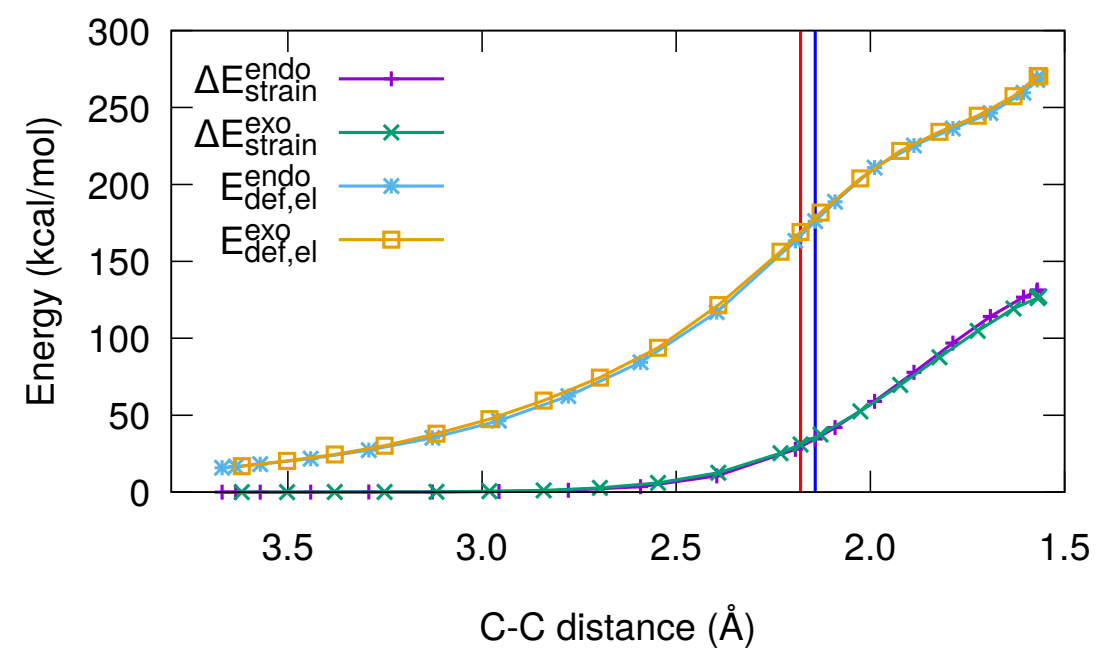

Figure 13: Geometric $\left(\Delta E_{\text {strain }}\right)$ and electronic $\left(E_{\text {def,el }}\right)$ deformation energies for the reaction between cyclopentadiene (A) and cyclopropenone (B) along the IRC for the endo and exo pathways. The blue and red lines mark the position of the endo and exo transition states, respectively.

exo curve below the endo one. Moreover, $V_{\mathrm{cl}}$ changes to destabilizing earlier (at $R \approx 2.5 \AA$ ) for the endo than for the exo (at $R \approx 2.1 \AA$ ) reaction, so that it stabilizes the exo TS (by $0.4 \mathrm{kcal} / \mathrm{mol}$ ) and destabilizes the endo one (by $0.5 \mathrm{kcal} / \mathrm{mol}$ ). The electrostatic interaction ceases to discriminate both routes past the TS.

We thus find here a rather more complex picture, one in which all elements play significant roles in determining the endo/exo preference. Now the geometric deformation does not fully predict the endo/exo ordering. Neither does the total deformation. Covalency effects, that might include SOIs, favor the exo route, particularly in the early phases. However, it is not until electrostatic effects are also added to the canvas when a full match is found at all points of the IRC.

\section{CONCLUSIONS}

We have examined in this paper the distortion/interaction or activation strain model in the light of the IQA energy decomposition. The ASM has been very successfully applied to understand a wide variety of chemical processes, which we here have illustrated through the 

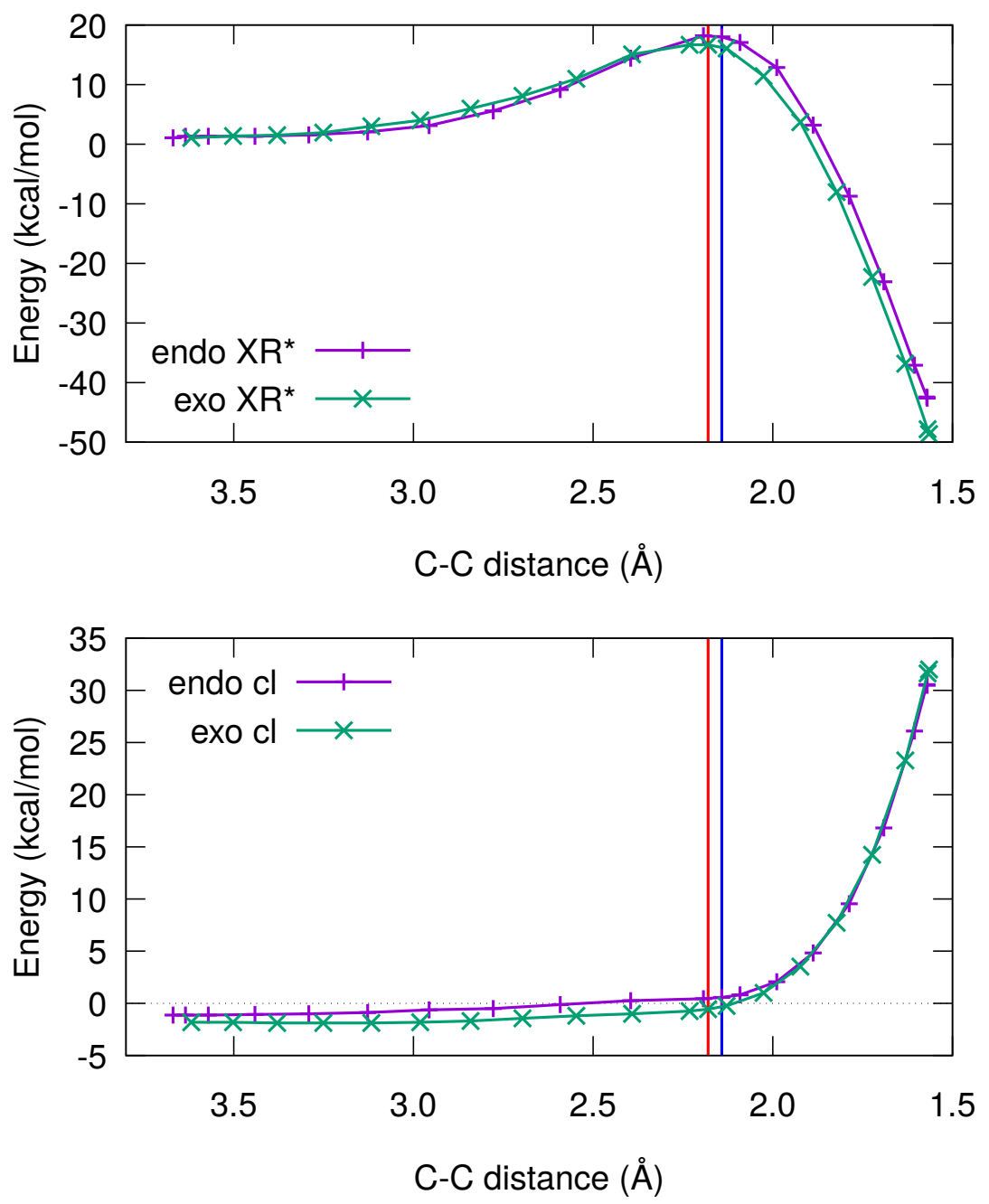

Figure 14: IQA interatomic energies $\mathrm{XR} \star=V_{\mathrm{xc}}^{A B}+E_{\mathrm{def}}^{A B}$ (top) and $V_{\mathrm{cl}}^{A B}$ (bottom) between cyclopentadiene (A) and cyclopropenone (B) along the IRC for the endo and exo reactions. The blue and red lines mark the position of the endo and exo transition states, respectively. 
endo/exo preference in simple Diels-Alder [4+2] cycloadditions. We have payed particular attention to the possible conceptual pitfalls in which one may fall when trying to find an "explanation" for a certain behavior from a sum of terms which may evolve differently upon the introduction of a perturbation (as the presence of another molecule) in a system.

The ASM divides the energy at any particular step during a process into a strain energy, the cost of geometrically deforming the isolated equilibrium fragments into their interacting structures, and everything else, which is called the interaction energy. During a reaction, for instance, the latter is generally stabilizing, so nothing else but the strain may "cause" any existing barrier. As it has been pointed out, both when a further EDA or IQA decomposition is made of the ASM interaction, further destabilizing terms (like the EDA Pauli repulsion, or the IQA electronic deformation) appear, which have been shown to play non-negligible roles. "Explaining" any interesting behavior exclusively in terms of $\Delta E_{\text {strain }}$ should thus be done with care.

As it has been done with EDA before, we have shown that IQA considerably enriches the plain ASM. Moreover, IQA does not use any intermediate non-stationary state as EDA does, and it may applied to a general fragmentation scheme with or without open-shells. Interestingly, the ASM picture regarding the endo rule stands still after an IQA scrutiny. For the endo-rule-following cyclopentadiene + maleic anhydride addition both secondary orbital interactions, which should lie hidden in the IQA exchange-correlation terms, as well as electrostatic interactions are shown to play very minor roles, and the endo preference can be justified easily from total deformation energies or only from their strain components. On the other hand, the endo-rule-violating cyclopentadiene + cyclopropanone reaction offers a much richer picture, where all strain, electrostatics and possibly SOIs are important. Only in the specific cases where one energetic component differs significantly among the different routes that are compared can it be justified to talk about the "cause" of a behavior, and always when this quantity is not related to a non-stationary intermediate state that may overshoot after relaxation.

Since IQA does not use, necessarily, non-stationary states, it is easier in our opinion to come to the relevant variables governing simple processes. Whenever some of the abovementioned conditions are not met, different rearrangements of the terms of the energy 
partitioning scheme, or the use of different relaxation mechanisms may lead to different interpretations.

\section{ACKNOWLEDGEMENT}

We thank the spanish MICINN, grant PGC2018-095953-B-I00, the FICyT, grant IDI/2018/000177, and the European Union FEDER funds for financial support. Finally, we are very grateful to Dr. Dimas Suárez for his careful reading of the manuscript and constructive comments.

\section{SUPPORTING INFORMATION}

Supporting information is available: cartesian coordinates along IRCs and full IQA tables for all the reactions examined.

\section{References}

1. R. B. Woodward and R. Hoffmann, Angew. Chem. Int. Ed., 1969, 8, 781-853.

2. K. Fukui, Acc. Chem. Res., 1971, 4, 57-64.

3. F. Weinhold and C. Landis, Valency and Bonding. A Natural Bond Orbital DonorAcceptor Perspective, Cambridge Univ. Press, 2005.

4. X. Krokidis, S. Noury and B. Silvi, J. Phys. Chem. A, 1997, 101, 7277-7282.

5. X. Krokidis, B. Silvi and M. Alikhani, Chem. Phys. Lett., 1998, 292, 35-45.

6. X. Krokidis, B. Silvi, C. Dezarnaud-Dandine and A. Sevin, New J. Chem., 1998, 22, 1341.

7. X. Krokidis, R. Vuilleumier, D. Borgis and B. Silvi, Mol. Phys., 1999, 96, 265-273.

8. R. A. Marcus, Ann. Rev. Phys. Chem., 1964, 15, 155-196.

9. P. Geerlings, F. D. Proft and W. Langenaeker, Chem. Rev., 2003, 103, 1793-1874. 
10. R. G. Parr and L. J. Bartolotti, J. Am.. Chem. Soc., 1982, 104, 3801-3803.

11. W. J. Mortier, K. V. Genechten and J. Gasteiger, J. Am.. Chem. Soc., 1985, 10\%, 829-835.

12. K. Szalewicz, Wiley Interdiscip. Rev. Comput. Mol. Sci., 2011, 2, 254-272.

13. W.-J. van Zeist and F. M. Bickelhaupt, Org. Biomol. Chem., 2010, 8, 3118.

14. I. Fernández and F. M. Bickelhaupt, Chem. Soc. Rev., 2014, 43, 4953-4967.

15. F. M. Bickelhaupt and K. N. Houk, Angew. Chem. Int. Ed., 2017, 56, 10070-10086.

16. T. Ziegler and A. Rauk, Inorg. Chem., 1979, 18, 1558-1565.

17. T. Ziegler and A. Rauk, Inorg. Chem., 1979, 18, 1755-1759.

18. T. Ziegler and A. Rauk, Theor. Chim. Acta, 1977, 46, 1-10.

19. K. Morokuma, J. Chem. Phys., 1971, 55, 1236-1244.

20. K. Morokuma, Acc. Chem. Res., 1977, 10, 294-300.

21. K. Kitaura and K. Morokuma, Int. J. Quantum Chem., 1976, 10, 325-340.

22. F. M. Bickelhaupt and E. J. Evert Jan Baerends, Rev. Comput. Chem., 2007, 15, 1-86.

23. L. Zhao, M. von Hopffgarten, D. M. Andrada and G. Frenking, Wiley Interdiscip. Rev. Comput. Mol. Sci., 2017, 8, e1345.

24. I. Fernández and G. Frenking, Eur. J. Org. Chem., 2018, 2019, 478-485.

25. M. P. Mitoraj, A. Michalak and T. Ziegler, J. Chem. Theory Comput., 2009, 5, 962-975.

26. W. H. E. Schwarz and H. Schmidbaur, Chem. Eur. J., 2012, 18, 4470-4479.

27. T. Bitter, K. Ruedenberg and W. H. E. Schwarz, J. Comput. Chem., 2006, 28, 411-422.

28. T. Bitter, S. G. Wang, K. Ruedenberg and W. H. E. Schwarz, Theor. Chem. Acc., 2010, $127,237-257$. 
29. S.-G. Wang and W. Schwarz, Angew. Chem. Int. Ed., 2009, 48, 3404-3415.

30. V. Pophristic and L. Goodman, Nature, 2001, 411, 565-568.

31. F. M. Bickelhaupt and E. J. Baerends, Angew. Chem. Int. Ed., 2003, 42, 4183-4188.

32. I. Fernández, G. Frenking and E. Uggerud, Chem. Eur. J., 2009, 15, 2166-2175.

33. A. Martín Pendás, M. A. Blanco and E. Francisco, J. Comput. Chem., 2009, 30, 98-109.

34. A. Martín Pendás, E. Francisco and J. Casals-Sainz, Intermolecular Interactions in Crystals: Fundamentals of Crystal Engineering, Royal Society of Chemistry, London, 1st edn, 2018, ch. 5, pp. 178-221.

35. M. A. Blanco, A. Martín Pendás and E. Francisco, J. Chem. Theory Comput., 2005, 1, 1096-1109.

36. E. Francisco, A. Martín Pendás and M. A. Blanco, J. Chem. Theory Comput., 2006, 2, 90-102.

37. I. Fernández and F. M. Bickelhaupt, J. Comput. Chem., 2013, 35, 371-376.

38. S. Sankararaman, Pericyclic Reactions - A Textbook: Reactions, Applications and Theory, Wiley-VCH, 2005.

39. K. Alder and G. Stein, Justus Liebigs Annalen der Chemie, 1934, 514, 1-33.

40. R. Hoffmann and R. B. Woodward, J. Am.. Chem. Soc., 1965, 87, 4388-4389.

41. R. B. Woodward and H. Baer, J. Am.. Chem. Soc., 1944, 66, 645-649.

42. A. Wassermann, J. Chem. Soc., 1935, 1511-1514.

43. D. Belluš, K. V. Bredow, H. Sauter and C. D. Weis, Helv. Chim. Acta, 1973, 56, 30043038.

44. C. Gonzalez and H. B. Schlegel, J. Phys. Chem., 1990, 94, 5523-5527.

45. W.-J. van Zeist and F. M. Bickelhaupt, Chem. Eur. J., 2010, 16, 5538-5541. 
46. R. F. W. Bader, Atoms in Molecules: A Quantum Theory, Clarendon Press, 1990.

47. A. Martín Pendás, M. A. Blanco and E. Francisco, J. Comput. Chem., 2006, 28, 161184.

48. A. Martín Pendás, E. Francisco, M. Blanco and C. Gatti, Chem. Eur. J., 2007, 13, 9362-9371.

49. P. Maxwell, A. Martín Pendás and P. L. A. Popelier, Phys. Chem. Chem. Phys., 2016, 18, 20986-21000.

50. E. Francisco, J. L. Casals-Sainz, T. Rocha-Rinza and A. Martín Pendás, Theor. Chem. Acc., 2016, 135, 170.

51. L. M. Stephenson, D. E. Smith and S. P. Current, J. Org. Chem., 1982, 47, 4170-4171.

52. T. H. Dunning, J. Chem. Phys., 1989, 90, 1007.

53. P. J. Stephens, F. J. Devlin, C. F. Chabalowski and M. J. Frisch, J. Phys. Chem., 1994, 98, 11623-11627.

54. A. D. Becke, J. Chem. Phys., 1993, 98, 1372-1377.

55. M. W. Schmidt, K. K. Baldridge, J. A. Boatz, S. T. Elbert, M. S. Gordon, J. H. Jensen, S. Koseki, N. Matsunaga, K. A. Nguyen, S. Su, T. L. Windus, M. Dupuis and J. A. Montgomery, J. Comput. Chem., 1993, 14, 1347-1363.

56. F. Weigend and R. Ahlrichs, Phys. Chem. Chem. Phys., 2005, 7, 3297-3305.

57. F. Weigend, J. Comput. Chem., 2008, 29, 167-175.

58. A. D. Becke, J. Chem. Phys., 1988, 88, 2547-2553.

59. B. Cordero, V. Gómez, A. E. Platero-Prats, M. Revés, J. Echeverría, E. Cremades, F. Barragán and S. Alvarez, Dalton Trans., 2008, 2832-2838. 
60. Q. Sun, T. C. Berkelbach, N. S. Blunt, G. H. Booth, S. Guo, Z. Li, J. Liu, J. D. McClain, E. R. Sayfutyarova, S. Sharma, S. Wouters and G. K.-L. Chan, Wiley Interdisciplinary Reviews: Computational Molecular Science, 2018, 8, e1340.

61. A. Martín Pendás and E. Francisco, Promolden. A QTAIM/IQA code (Avaliable from the authors upon request).

62. T. Sexton, E. Kraka and D. Cremer, J. Phys. Chem. A, 2016, 120, 1097-1111.

63. A. Martín Pendás, M. A. Blanco and E. Francisco, J. Chem. Phys., 2006, 125, 184112.

64. J. L. Casals-Sainz, A. Costales, E. Francisco and A. Martín Pendás, Molecules, 2019, 24, 2204.

65. C. Outeiral, M. A. Vincent, A. Martín Pendás and P. L. A. Popelier, Chem. Sci., 2018, 9, 5517-5529.

66. M. García-Revilla, P. L. A. Popelier, E. Francisco and A. Martín Pendás, J. Chem. Theory Comput., 2011, 7, 1704-1711.

67. A. Martin Pendás, J. L. Casals-Sainz and E. Francisco, Chem. Eur. J., 2018, 25, 309314. 